\title{
The Role of Dust in GRB Afterglows
}

\author{
Donald Q. Lamb \\ Department of Astronomy \& Astrophysics, University of Chicago, 5640 South Ellis \\ Avenue, Chicago, IL 60637
}

\begin{abstract}
We show that the clumpy structure of star-forming regions can naturally explain the fact that 50-70\% of GRB afterglows are optical "dark." We also show that dust echos from the GRB and its afterglow, produced by the clumpy structure of the star-forming region in which the GRB occurs, can lead to temporal variability and peaks in the NIR, optical, and UV lightcurves of GRB afterglows.
\end{abstract}

\section{Introduction}

There is increasing evidence that the long gamma-ray bursts (GRBs) are associated with galaxies undergoing copious star formation, and occur near or in the star-forming regions of these galaxies (see, e.g., [1] for a discussion of this evidence). Star-forming regions contain large amounts of dust that can extinguish the optical and UV light of GRB afterglows. Indeed, no optical afterglows have been detected for $60-70 \%$ of the long GRBs. Some of these failures may be due to the relatively large size of the GRB error box, or to a delay in observing the error box. Some may be because the GRB lies at a very high redshift, and the Lyman limit lies longward of the optical band $[2,3]$. However, the majority of the failures are most likely because the optical afterglow is faint or absent due to its extinction by dust in the host galaxy of the GRB.

We show that the clumpy structure of star-forming regions can naturally explain the statistics of optically "dark" GRB afterglows. We also show that dust echos from the GRB and its afterglow, produced by the clumpy structure of the star-forming region in which the GRB occurs, can lead to temporal variability and peaks in the NIR, optical, and UV lightcurves of GRB afterglows.

\section{Structure of Star-Forming Regions}

Star-forming regions are thought to be clumpy, with dense dust clouds embedded in a much less dense intercloud medium. A simple model of star-forming regions can therefore be characterized by three parameters: (1) the amount of dust equivalent to a radial optical depth $\tau_{H}$ of a homogeneous uniform density medium, the volume filling factor $f$ of the dust clumps, and the density contrast $k_{1} / k_{2}$ of the clumps relative to the interclump medium [4,5]. In this model, the dense dust clumps form connected structures, as is true in real star-forming regions. 


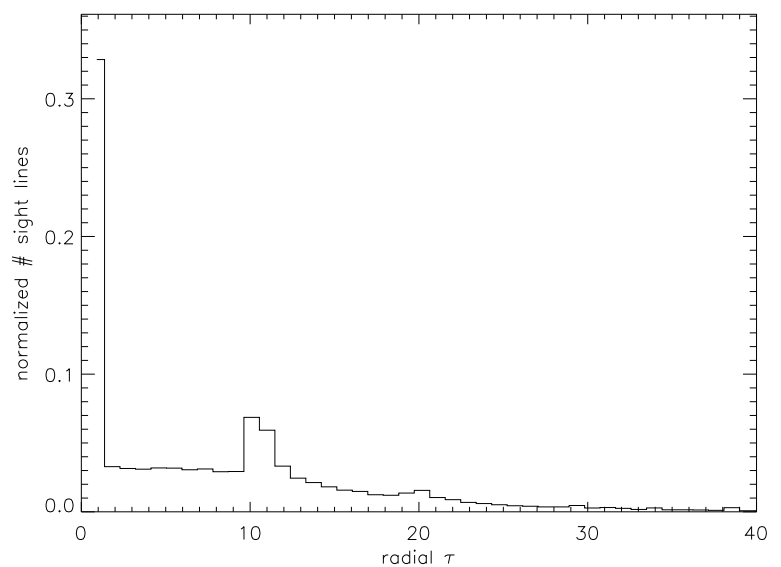

Fig. 1. Distribution of optical depths, as expereinced by random photons emitted isotropically by a central source in a clumpy medium with an amount of dust equivalent to a radial optical depth $\tau_{H}=10$ in a homogeneous uniform density medium, a volume filling factor $f=0.10$ of the clumps, and a density contrast $k_{1} / k_{2}=100$ between the clumps and the interclump medium. From [4].

Figure 1 shows the of a Monte Carlo calculation of the distribution of optical depths for random lines of sight (LOS) from the center of a clumpy star-forming region to an external observer. The parameters of this particular calculation are $\tau_{H}=10, f=0.10, k_{1} / k_{2}=100$, and the number $N^{3}$ of spatial bins is $20^{3}$ [4]. These parameter values lead to results that are consistent with observations of star-forming regions in the Milky Way. Figure 1 shows that 35\% of LOS have optical depths $\tau_{\text {obs }}=1$ (the minimum value possible in this particular model), while the remaining $65 \%$ of LOS have $\tau_{\text {obs }} \gg 1$. The distribution of optical depths can be understood as follows. A substantial fraction of the photons emitted by the central source do not encounter a dense clump; these photons correspond to the LOS with $\tau_{\text {obs }}=1$. The remaining photons emitted by the central source encounter one or more clumps and experience $\tau_{\text {obs }} \gg 1$. The distribution of $\tau_{\text {obs }}$ in this model is consistent with the statistics of GRB optical afterglows, 50-70\% of which are optically "dark."

\section{Dust Echos}

Dust echos from the clumpy structure of the star-forming region can also produce variability and peaks $1^{\mathrm{d}}-30^{\mathrm{d}}$ after the GRB in the NIR, optical, and UV lightcurves of GRB afterglows. As an illustrative example, we have calculated the optical light curve produced by the dust echo from a single clump at a substantial distance from the burst source but along the LOS from the burst source to the observer. The delay time $\delta t$ between the GRB and the echo is characterized by $\delta t=(1+z) R_{\perp, \min }^{2} / c D$, where $D$ is the distance between the GRB and the dust cloud and $R_{\perp, \min }$ is the minimum of the perpendicular extent of the dust cloud $R_{\perp, \text { cloud }}, \theta_{\text {jet }}(t) D$, and $\theta_{\text {forward }} D$. Here $\theta_{\text {jet }}(t)$ is the half opening angle of the afterglow jet and $\theta_{\text {forward }}$ is the half angular width $\left(\approx 10^{\circ}-20^{\circ}\right)$ of the forward scattering peak for scattering by dust grains.

The amplitude $A$ of the dust echo relative to the direct light from the afterglow is a function of the albedo $a$ of the dust, the scattering phase function 

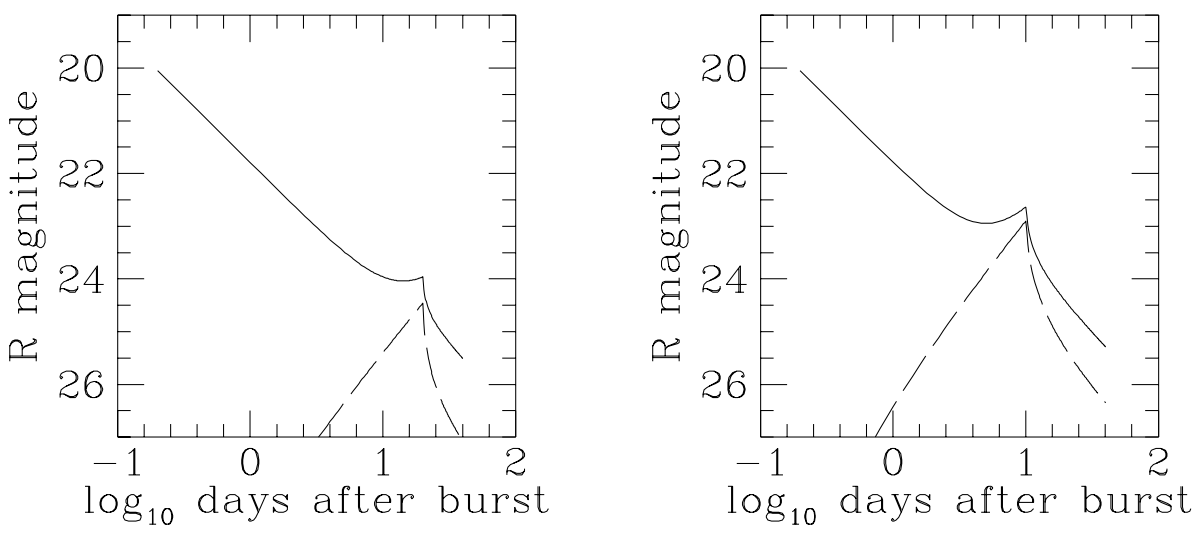

Fig. 2. Contribution to the observed R-magnitude from the light forward-scattered by the dust cloud (dashed line), and the sum of this light and the light seen directly from the point-like source (solid curve) for two sets of parameters of the simple model described in the text. Left: $\delta t=20$ days $A=0.2, \epsilon=0.1$ days. Right: $\delta t=10$ days $A=0.5, \epsilon=0.1$ days.

$\Phi(\theta)$, and the optical depth $\tau_{\text {clump }}$ of the dust clump, all of which are wavelength dependent. For forward scattering, which we assume here, $\Phi(\theta) \approx 1$ [6]. Then in the limiting cases of small and large optical depths, $A \approx a \tau_{\text {clump }}$ and $A \approx a$, respectively. The prominence of the dust echo also depends on the time $\epsilon$ after the GRB that the afterglow begins.

Figure 2 shows the dust echo from a single clump along the LOS from the burst source to the observer, assuming $\tau_{\text {clump }}<1$. In the two cases shown, the rate of temporal decline of the GRB afterglow was taken to be a power law with $b=1$. Most GRB afterglows decline more rapidly with time (i.e., $b=1.3-2.25$ ). The dust echo is more prominent for afterglows that decline more rapidly, since the contrast between the direct light from the afterglow and the echo - which reflects the brightness of the afterglow at an earlier time - is then larger. Thus the examples we have shown are conservative.

Studies of the temporal variability of the NIR, optical, and UV lightcurves of the afterglows of GRBs may allow "reverberation mapping" of the structure of the star-forming regions in which GRBs occur.

\section{References}

1. Lamb, D. Q. 2000, Phys. Reports, 333, 505

2. Fruchter, A. S. 1999, ApJ, 512, L1

3. Lamb, D. Q. \& Reichart, D. E. 2000, ApJ, 536, 1

4. Witt, A. N. \& Gordon, K. D. 1996, ApJ, 463, 681

5. Witt, A. N. \& Gordon, K. D. 2000, ApJ, 528, 799

6. van de Hulst, H. C. 1957, Light Scattering by Small Particles (New York: Wiley), Chapter 9 